\title{
Chromatographic Separation and Identification of Sildenafil and Yohimbine Analogues Illegally Added in Herbal Supplements
}

\author{
Hakan Göker*, Maksut Coşkun and Gülgün Ayhan-Kılcıgil \\ Central Instrumental Analysis II Laboratory, Faculty of Pharmacy, Ankara University, \\ Tandogan, Ankara, \\ Turkey
}

\section{Introduction}

Herbal medicines are major source of aphrodisiacs and have been used worldwide for thousands of years by different cultures and civilizations. Recently, consumption of dietary supplements has been becoming more popular around the world. Unfortunately, the adulteration of dietary supplements with undeclared synthetic chemical compounds is steadily increasing according to the literature. Some herbal products advertised as "all natural" have in contrast been found to contain synthetic PDE-5 inhibitors. There are currently three PDE5 inhibitors Sildenafil (Langtry \& Markham, 1999) (Viagra; Pfizer, New York, US), Tadalafil (Meuleman, 2003) (Cialis; Eli Lilly, Indianapolis, US), and Vardenafil (Keating \& Scott, 2003) (Levitra; Bayer Pharmaceuticals Co, Wuppertal, Germany), approved worldwide for the treatment of male erectile dysfunction, further two agents Udenafil (Salem et al., 2006) (Zydena; Dong-A PharmTech Co, Korean), Mirodenafil (Jung, 2008) (Mvix, Life Science R\&D Center of SK chemical, Beijing, Tianjin, Shanghai) were licensed only in Korea. They produce vascular smooth muscle relaxation, promote penile blood flow, and hence, induce erection. These kinds of commercially available herbal aphrodisiac products have been spiked with the above-mentioned legal drugs, but also with their analogues, which have not been subjected to formal pharmacokinetic or other pharmacological testing in either humans or animals.

The practice of self-medication by an increasing number of patients, the incessant aggressive advertising of these herbal aphrodisiacs, the invasion of the medicinal market with uncontrolled dietary supplements and the absence of real directives amplifies the potential health hazards to the community. Since the sildenafil is an chemical, it must not been found in any foodstuffs, but an increasing number of sildenafil analogues have been discovered in dietary and herbal supplements even in soft drinks, this number is steadily increasing and some time their types are unknown and necrosis not observed instead of paracetamol. Hence, it is prudent to test the safety and efficacy thus might have unknown and harmful side-effects. Structural analogues are also synthetic chemicals with slightly altered chemical structures and have similar erectile effects on the body. Nevertheless, it is not uncommon

${ }^{*}$ Corresponding Author 
for chemicals with similar structures to possess slightly or entirely different before any new chemical is licensed as drug for human use. This testing process is lengthy and costly; on average, it takes 9.5 years and costs US\$800 million to license a new drug. Many drug analogues, without the aforementioned drug testing process, are available for human consumption properties. Phenacetin, structurally similar to paracetamol, has been associated with renal papillary necrosis not observed with paracetamol (Poon, 2007). Many drug analogues, without the aforementioned drug testing process, are avaliable for human comsuption via different channels. Examples include analogues of psychoactive drugs, anabolic steroids, and Sibutramine which was one of the most abused compound as antiobesity drugs.

The most commonly reported side effects of sildenafil are headaches, flushing of the face, upset stomach and nasal congestion. We met a dieatery supplement having combination of sildenafil and paracetamol, probably in order to prevent headeache caused by sildenafil. Other side effects include sensitivity to light, blurred vision, urinary tract infection, diarrhea and dizziness. The main problem with sildenafil and analogues are that they interact with many other medications. They can rapidly decrease blood pressure up to dangerously low. They can interact with nitrates such as nitrogliserin, which are often prescribed to heart patients. Sildenafil has not to be administrated to patients with heart problems taking nitrate medications because of the severe potentiation of vasodilatory effects.

It is well known, the first developed and consequently the most famous phosphodiesterase inhibitor is Sildenafil (Langtry \& Markham, 1999) approved by the FDA in early April 1998. Novel PDE5 inhibitor, Lodenafil carbonate, breaks down in the body to form two molecules of the active drug lodenafil. This formulation has higher oral bioavailability than the parent drug (Toque et al., 2008) Fig 1.<smiles>CCCc1nn(C)c2c(=O)[nH]c(-c3cc(S(=O)(=O)N4CCN(CCOC(=O)OCCN5CCN(S(=O)(=O)c6ccc(OCC)c(-c7nc8c(CCC)nn(C)c8c(=O)[nH]7)c6)CC5)CC4)ccc3OCC)nc12</smiles><smiles>CCCc1nn(C)c2c(=O)[nH]c(-c3cc(S(=O)(=O)N4CCN(CCO)CC4)ccc3OCC)nc12</smiles>

Lodenafil

Fig. 1. Prodrug lodenafil carbonate. 
Avanafil was discovered by pharmaceutical developer "Vivus" as PDE5 inhibitors, the safety and efficacy for erectile dysfunction, a possible lunch in 2012. It has a particular advantage over its potential competitors, the effects are demonstrable very quickly (in 15 minutes or less) (Bell \& Palmer, 2011) Fig 2.<smiles>COc1ccc(CNc2nc(N3CCCC3CO)ncc2C(=O)NCc2ncccn2)cc1Cl</smiles>

Fig. 2. Avanafil.

The one of the last discovered illegal PDE-5 inhibitor is called as "Acetylvardenafil" which was found in dieatery supplement known as MEGATON® in USA (Lee et al., 2011). Sulfonyl group of Vardenafil was substituted by an acetyl group Fig 3(a) .

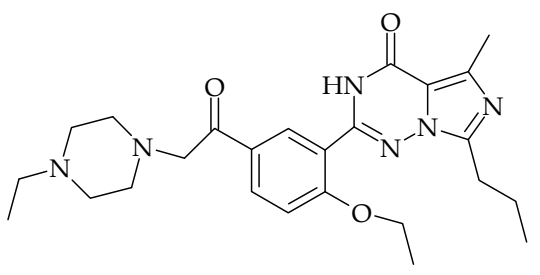

(a)<smiles>CCCc1nc(C)c2c(=O)[nH]c(-c3ccccc3OCC)nn12</smiles>

(b)<smiles>CCCc1nn(C)c2c(=O)[nH]c(-c3cc(C(C)=O)ccc3OCC)nc12</smiles>

(c)

Fig. 3. (a) Acetylvardenafil; (b) Desulfovardenafil; (c) Gendenafil.

Another new analogue of vardenafil, Desulfovardenafil, in which the N-ethylpiperazine ring and the sulphonyl group were removed from the vardenafil structure, was identified for enhancing erectile function in herbal health product marketed, namely Power58 Platinum (Lai et al., 2007, Lam, 2007) Fig 3(b). New sildenafil analogues Gendenafil had an acetyl group instead of sulfonyl- $N$-methylpiperazine moiety was determined as 5-[2-ethoxy5-acetyl-phenyl]-1-methyl-3-n-propyl-1,6-dihydro-7H-pyrazolo[4,3- $d$ ]pyrimidin-7-one (Lin et al., 2008) Fig3(c). Other vardenafil analogue was found to be added illegally into a dietary supplement marketed for male erectile dysfunction (MED). Its structure was determined as 2-(2-ethoxyphenyl)-5-methyl-7-propyl-imidazo[5,1-f][1,2,4]triazin-4(3H)-one and called "Piperidenafil or Piperidino vardenafil or trivial name Pseudovardenafil" (Park et al., 2007; Lai et al., 2007b) Fig 4(a).

Very recently, two new analogues of sildenafil in which the piperazine ring and the sulfonyl group were replaced by a piperazinone and a hydroxyethyl structure, respectively were isolated from a herbal product in Germany. Based on the piperazinone structure, the compounds were named Piperazinonafil in Fig 4(b) and Isopiperazinonafil (Wollein et al., 2011) in Fig 4(c). 
<smiles>CCCc1nc(C)c2c(=O)[nH]c(-c3cc(C(=O)CN4CCCCC4)ccc3OCC)nn12</smiles>

(a)<smiles>CCCc1nn(C)c2c(=O)[nH]c(-c3cc(C(O)CN4CCN(CC)CC4)ccc3OCC)nc12</smiles>

(b)<smiles>CCCc1nn(C)c2c(=O)[nH]c(-c3cc(CC(O)N4CCN(CC)CC4)ccc3OCC)nc12</smiles>

(c)

Fig. 4. (a) Piperidenafil; (b) Piperazinonafil; (c) Isopiperazinonafil.

Another sildenafil analogue was detected from a health supplement claimed for human MED, the structure of this new analogue was characterized as dithiodesmethylcarbodenafil containing 2 thiocarbonyl groups instead of 2 carbonyl groups, and 4-methyl substitution on the piperazine ring, rather than 4-ethyl substitution when compared to sildenafil (Ge et al., 2011) Fig. 5(a).

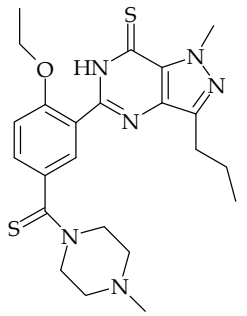

(a)

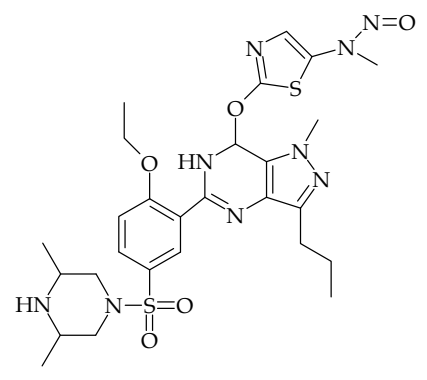

(b)

Fig. 5. (a) Dithio-desmethylcarbodenafil; (b) Nitroso-prodenafil.

Other new unapproved analogue of sildenafil was detected in capsules of a herbal dietary supplement promoted as a libido enhancing product. This is the first time a PDE-5 inhibitor and a potential NO donor were identified in one molecule. A hydrolysis experiment showed that the new analogue was a prodrug of aildenafil and was therefore named nitrosoprodenafil (Venhuis et al., 2011) Fig 5(b). Both PDE-5 inhibitors and nitrosamines cause vasodilatation by increasing levels of NO. To their coincidental use is warned against because it may cause a fatal drop in blood pressure. In addition, nitrosamines are known carcinogens. The findings indicate the dangerous level of advancement in medicinal chemistry by producers of unapproved drugs.

Tadalafil (Cialis $\left.{ }^{\circledR}\right)$ was approved in 2003 by the FDA as the third phosphodiesterase type 5 enzyme (PDE-5) inhibitor to treat MED (Meuleman, 2003). Then, different tadalafil analogues have been found as adulterants in illegal products. Hasegawa et al. detected Noctyl-nortadalafil Fig 6(a) together with cyclopentynafil Fig 6(b) in dietary supplement (Hasegawa et al., 2008). Both of them are the first compounds reported to be new tadalafil and sildenafil analogues. 
<smiles>CCCCCCCCN1CC(=O)N2C(Cc3c([nH]c4ccccc34)C2c2ccc3c(c2)OCO3)C1=O</smiles>

(a)<smiles>CCCc1nn(C)c2c(=O)[nH]c(-c3cc(S(=O)(=O)N4CCN(C5CCCC5)CC4)ccc3OCC)nc12</smiles>

(b)

Fig. 6. (a) N-octylnor-tadalafil; (b) Cyclopentynafil.

In Taiwan, one of the dietary supplement which was claimed on the treatment of male erectile dysfunction was firstly screened in 2009 and Tadalafil and its doctored version was newly identified. Since it is having amino group instead of methyl in tadalafil it was named as aminotadalafil (Zou et al., 2006; Lin et al., 2009) Table-1. This compound has two asymmetric carbons, theoritically two pairs of enantiomers exist. The chromatographic separation of its stereoisomers was reported by using chiral LC-MS (Kurita et al., 2008). Using this method, RR-Aminotadalafil and SR-Aminotadalafil were detected in some health food. In addition, an interaction product of aminotadalafil was isolated from an illegal health food product. The structure of the interaction product was elucidated and unknown compound was characterized as condensation product of aminotadalafil and hydroxymethylfuraldehyde and is probably the result of a drug-excipient incompatibility (Häberli et al., 2010) Fig 7.<smiles>O=C1C2Cc3c([nH]c4ccccc34)C(c3ccc4c(c3)OCO4)N2C(=O)CN1/N=C/c1ccc(CO)o1</smiles>

Fig. 7. Condensation product of aminotadalafil and hydroxymethylfuraldehyde.

Last flash development for the treatment of MED is discovering of Zoraxel (RX-10100) by Rexahn Pharmaceutical company (Albersen et al., 2010). Zoraxel is containing clavulanic acid that is centrally acting in the CNS and may be a more effective MED treatment for patients who are responsive or unresponsive to PDE- 5 inhibitors. It is being developed as an orally administered, on-demand tablet to treat sexual dysfunction, and has extensive and well-established safety in humans. For the future, it is being expected, Zoraxel will be on to worldwide best-selling drug. 
In our central instrumental analysis laboratory, we also try to detect these commercially available supplements (which are sent by the Ministry of Food Agriculture and Livestock of Turkey before it grants a license for import to Turkey) whether they possess new or old sildenafil analogues by using high-performance liquid chromatography with diode array detection and mass spectrometry (HPLC-DAD-MS) and nuclear magnetic resonance (NMR) analyses, NMR is the only analytical technique which provides full structural information from novel compounds, acquisition of MS data or comparison of retention times may not be sufficient, in this case, LC-MS/NMR allowed to identify the adulterants without any need for references (Kesting et al., 2010) this means that these kind of analogues are not easy to detect by ordinary laboratory methods, we have identified the listed analogues are given in Table 1-2 up to date. With the aim of evaluating the potential risks of commercialized aphrodisiac products on consumer health, the aim of present work is to investigate simple HPLC-MS method and NMR data of synthetic and natural analogues of aphrodisiacs.

Table-1 shows the determination of some sildenafil and tadalafil analogues and Dapoxetin $\mathrm{HCl}$, in commercially available health supplements in Turkey. Their formulas, ${ }^{1} \mathrm{H}-{ }^{13} \mathrm{C}-\mathrm{NMR}$ spectra, ESI $(+)$ m/e values and their LC chromatograms are given in Table 1 and Fig 8, 9, respectively. Fig 9 shows the good separation of caffeine, some sildenafil analogues and

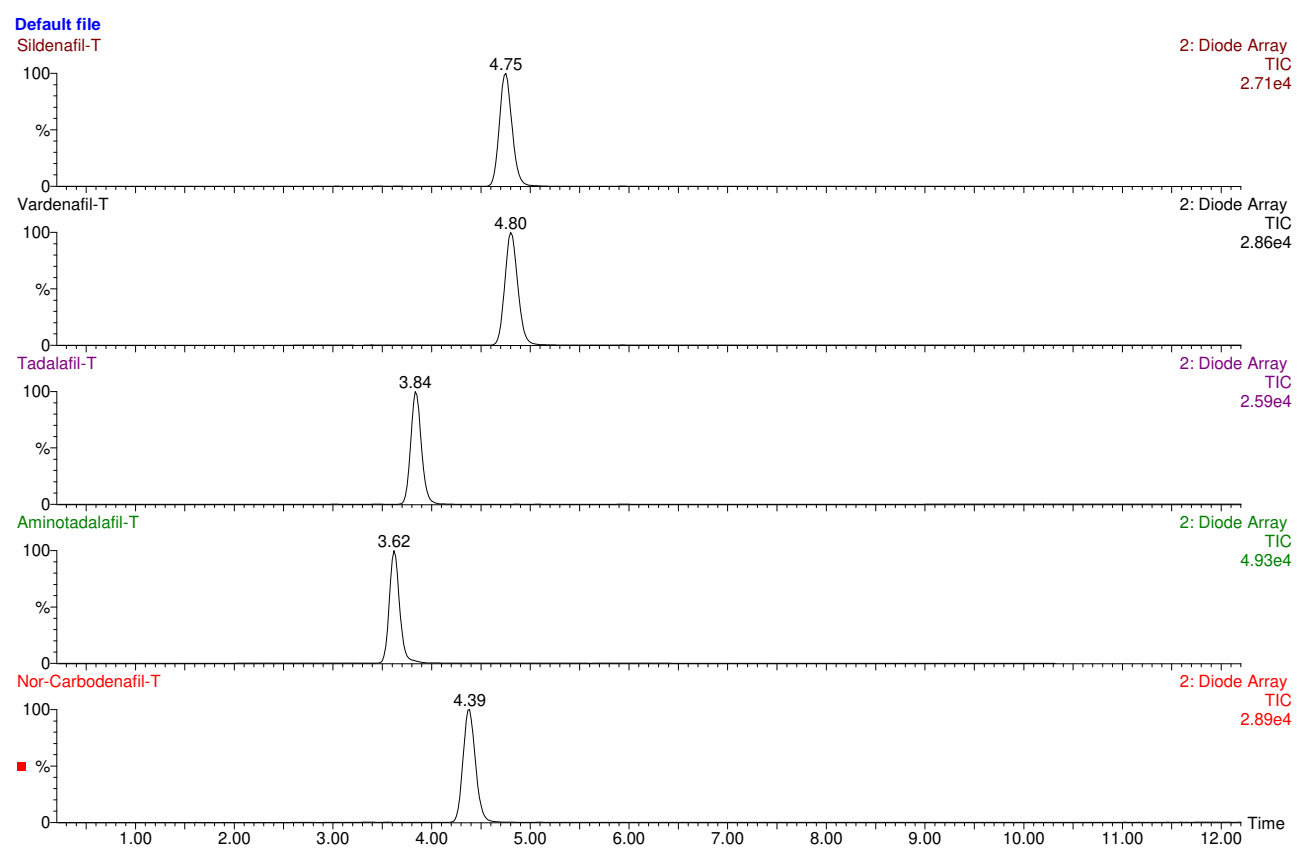

Fig. 8. HPLC chromatogram of 4.75 (Sildenafil), 4.80 (Vardenafil), 3.84 (Tadalafil), 3.62 (Aminotadalafil), 4.39 (Nor-carbodenafil) (RT: Retention times as min). 


\begin{tabular}{|c|c|c|c|}
\hline Compound name & $\begin{array}{c}\text { M.w. } \\
E S I(+) \mathrm{m} / \mathrm{z}\end{array}$ & Formulas & 1H-NMR $\delta$ ppm \\
\hline $\begin{array}{c}\text { Sildenafil citrate } \\
\text { (Langtry \& Markham, 1999) } \\
\mathrm{C}_{22} \mathrm{H}_{30} \mathrm{~N}_{6} \mathrm{O}_{4} \mathrm{~S} . \mathrm{C}_{6} \mathrm{H}_{8} \mathrm{O}_{7}\end{array}$ & $\begin{array}{c}474 \\
475(M+H)\end{array}$ & & $\begin{array}{l}\left(\mathrm{CD}_{3} \mathrm{OD}\right): 0.99(\mathrm{t}, 3 \mathrm{H}), 1.46(\mathrm{t}, 3 \mathrm{H}), 1.79(\mathrm{~m}, 2 \mathrm{H}), 2.59(\mathrm{~s}, 3 \mathrm{H}), 2.64, \\
2.72,2.78,2.82 \text { (citrate prot.), } 2.87(\mathrm{t}, 2 \mathrm{H}), 2.97(\mathrm{br} . \mathrm{t}, 4 \mathrm{H}), 3.22 \text { (br.s, } \\
4 \mathrm{H}), 4.23(\mathrm{~s}, 3 \mathrm{H}), 4.29(\mathrm{q}, 2 \mathrm{H}), 7.37(\mathrm{~d}, \mathrm{~J}=8.8 \mathrm{~Hz}, 1 \mathrm{H}), 7.9(\mathrm{dd}, \mathrm{J}=8.8, \\
2.4 \mathrm{~Hz}, 1 \mathrm{H}), 8.14(\mathrm{~d}, \mathrm{~J}=2.4 \mathrm{~Hz}, 1 \mathrm{H})\end{array}$ \\
\hline $\begin{array}{c}\text { Tadalafil } \\
\text { (Meuleman, 2003) } \\
\mathrm{C}_{22} \mathrm{H}_{19} \mathrm{~N}_{3} \mathrm{O}_{4}\end{array}$ & $\begin{array}{c}389 \\
390(M+H)\end{array}$ & & \begin{tabular}{|l|}
$\left(\mathrm{CD}_{3} \mathrm{OD}\right): 2.93(\mathrm{~s}, 3 \mathrm{H}), 3.05 \& 3.1(\mathrm{dd}, 11.6,1.2 \mathrm{~Hz} \& 11.6,1.2 \mathrm{~Hz}$, \\
$1 \mathrm{H}), 3.66(\mathrm{dd}, \mathrm{J}=16.0,4.4 \mathrm{~Hz}, 1 \mathrm{H}), 3.97(\mathrm{~d}, \mathrm{~J}=17.6 \mathrm{~Hz}, 1 \mathrm{H}), 4.20(\mathrm{dd}$, \\
$\mathrm{J}=17.6,1.6 \mathrm{~Hz}, 1 \mathrm{H}), 4.43(\mathrm{dd}, \mathrm{J}=11.2,4 \mathrm{~Hz}, 1 \mathrm{H}), 5.84(\mathrm{dd}, \mathrm{J}=5.6,1.2 \mathrm{~Hz}$, \\
$2 \mathrm{H}), 6.17(\mathrm{~s}, 1 \mathrm{H}), 6.67(\mathrm{~d}, \mathrm{~J}=7.6 \mathrm{~Hz}, 1 \mathrm{H}), 6.79(\mathrm{~d}, \mathrm{~J}=1.6 \mathrm{~Hz}, 1 \mathrm{H}), 6.82$ \\
$(\mathrm{dd}, \mathrm{J}=8.0,1.6 \mathrm{~Hz}, 1 \mathrm{H}), 7.01(\mathrm{td}, \mathrm{J}=6.8,0.8 \mathrm{~Hz}, 1 \mathrm{H}), 7.06(\mathrm{td}, \mathrm{J}=7.6$, \\
$1.2 \mathrm{~Hz}, 1 \mathrm{H}), 7.25(\mathrm{~d}, \mathrm{~J}=8 \mathrm{~Hz}, 1 \mathrm{H}), 7.51(\mathrm{~d}, \mathrm{~J}=8 \mathrm{~Hz}, 1 \mathrm{H})$.
\end{tabular} \\
\hline $\begin{array}{l}\text { Vardenafil HCl } \\
\text { (Keating \& Scott, 2003) } \\
\mathrm{C}_{23} \mathrm{H}_{32} \mathrm{~N}_{6} \mathrm{O}_{4} \mathrm{~S} . \mathrm{HCl}\end{array}$ & $\begin{array}{c}488 \\
489(M+H)\end{array}$ & & $\begin{array}{l}\left(\mathrm{CD}_{3} \mathrm{OD}\right): 0.98(\mathrm{t}, 3 \mathrm{H}), 1.32(\mathrm{t}, 3 \mathrm{H}), 1.45(\mathrm{t}, 3 \mathrm{H}), 1.82(\mathrm{~m}, 2 \mathrm{H}), 2.52 \\
(\mathrm{~s}, 3 \mathrm{H}), 2.96(\mathrm{t}, 2 \mathrm{H}), 3.19(\mathrm{q}, 2 \mathrm{H}), 3.3(\mathrm{br} . \mathrm{s}, 8 \mathrm{H}), 4.29(\mathrm{q}, 2 \mathrm{H}), 7.41(\mathrm{~d}, \\
\mathrm{J}=8.8 \mathrm{~Hz}, 1 \mathrm{H}), 7.98(\mathrm{td}, \mathrm{J}=9.2,1.6 \mathrm{~Hz}, 1 \mathrm{H}), 8.05(\mathrm{~d}, \mathrm{~J}=2 \mathrm{~Hz}, 1 \mathrm{H}) .\end{array}$ \\
\hline $\begin{array}{l}\text { Udenafil } \\
\text { (Salem et al., 2006) } \\
\mathrm{C}_{25} \mathrm{H}_{36} \mathrm{~N}_{6} \mathrm{O}_{4} \mathrm{~S}\end{array}$ & $\begin{array}{c}516 \\
517(M+H)\end{array}$ & & $\begin{array}{l}\left(\mathrm{CD}_{3} \mathrm{OD}\right): 0.99(\mathrm{t}, 3 \mathrm{H}), 1.04(\mathrm{t}, 3 \mathrm{H}), 1.36(\mathrm{~m}, 2 \mathrm{H}), 1.69-2.05(\mathrm{~m}, 8 \mathrm{H}) \\
2.18(\mathrm{~m}, 2 \mathrm{H}), 2.27(\mathrm{~s}, 3 \mathrm{H}), 2.85-3.05(\mathrm{~m}, 6 \mathrm{H}), 4.18(\mathrm{t}, 2 \mathrm{H}), 4.23(\mathrm{~s}, 3 \mathrm{H}) \\
7.34(\mathrm{~d}, \mathrm{~J}=8.4 \mathrm{~Hz}, 1 \mathrm{H}), 7.96(\mathrm{dd}, \mathrm{J}=8.4,2.4 \mathrm{~Hz}, 1 \mathrm{H}), 8.25(\mathrm{dd}, \mathrm{J}=2.4 \\
0.8 \mathrm{~Hz}, 1 \mathrm{H}) .\end{array}$ \\
\hline $\begin{array}{c}\text { Aminotadalafil } \\
\text { (Zou et al., 2006; Kurita, 2008, Lin et } \\
\text { al., 2009) } \\
\mathrm{C}_{21} \mathrm{H}_{18} \mathrm{~N}_{4} \mathrm{O}_{4} \\
\text { The mixture of isomers }\end{array}$ & $\begin{array}{c}390 \\
391(M+H)\end{array}$ & & $\begin{array}{l}\left(\mathrm{CD}_{3} \mathrm{OD}\right): 3.11 \& 3.14(\mathrm{dd}, 14.4,1.2 \mathrm{~Hz} \& 11.6,0.8 \mathrm{~Hz}, 1 \mathrm{H}), 3.69(\mathrm{dd}, \\
\mathrm{J}=16.0,4.4 \mathrm{~Hz}, 1 \mathrm{H}), 4.11(\mathrm{~d}, \mathrm{~J}=17.6 \mathrm{~Hz}, 1 \mathrm{H}), \quad 4.29(\mathrm{dd}, \mathrm{J}=17.6,2 \mathrm{~Hz}, \\
\mathrm{lH}), 4.43(\mathrm{dd}, \mathrm{J}=11.2,3.6 \mathrm{~Hz}, 1 \mathrm{H}), 5.84(\mathrm{dd}, \mathrm{J}=5.6,1.2 \mathrm{~Hz}, 2 \mathrm{H}), 6.14 \\
(\mathrm{~s}, 1 \mathrm{H}), 6.67(\mathrm{~d}, \mathrm{~J}=7.6 \mathrm{~Hz}, 1 \mathrm{H}), 6.80(\mathrm{~d}, \mathrm{~J}=2 \mathrm{~Hz}, 1 \mathrm{H}), 6.84(\mathrm{dd}, \mathrm{J}=8.2, \\
1.6 \mathrm{~Hz}, 1 \mathrm{H}), 7.01(\mathrm{td}, \mathrm{J}=6.8,0.8 \mathrm{~Hz}, 1 \mathrm{H}), 7.06(\mathrm{td}, \mathrm{J}=7.6,1.6 \mathrm{~Hz}, 1 \mathrm{H}) \\
7.24(\mathrm{~d}, \mathrm{~J}=8 \mathrm{~Hz}, 1 \mathrm{H}), 7.51(\mathrm{~d}, \mathrm{~J}=8 \mathrm{~Hz}, 1 \mathrm{H}) .\end{array}$ \\
\hline $\begin{array}{l}\text { Thiosildenafil } \\
\text { (Zou et al., 2008) } \\
\mathrm{C}_{22} \mathrm{H}_{30} \mathrm{~N}_{6} \mathrm{O}_{3} \mathrm{~S}_{2}\end{array}$ & $\begin{array}{c}490 \\
491(M+H)\end{array}$ & & $\begin{array}{l}\left(\mathrm{CD}_{3} \mathrm{OD}\right):{ }^{1} \mathrm{H}-\mathrm{NMR} 1.0(\mathrm{t}, 3 \mathrm{H}), 1.54(\mathrm{t}, 3 \mathrm{H}), 1.82(\mathrm{~m}, 2 \mathrm{H}), 2.26(\mathrm{~s}, \\
3 \mathrm{H}), 2.51(\mathrm{br} . \mathrm{t}, 4 \mathrm{H}), 2.9(\mathrm{t}, 2 \mathrm{H}), 3.06(\mathrm{br} . \mathrm{s}, 4 \mathrm{H}), 4.34(\mathrm{q}, 2 \mathrm{H}), 4.48(\mathrm{~s} \\
3 \mathrm{H}), 7.39(\mathrm{~d}, \mathrm{~J}=8.4 \mathrm{~Hz}, 1 \mathrm{H}), 7.9(\mathrm{dd}, \mathrm{J}=8.4,2.4 \mathrm{~Hz}, 1 \mathrm{H}), 8.33(\mathrm{~d} \\
\mathrm{J}=2.4 \mathrm{~Hz}, 1 \mathrm{H}) .\end{array}$ \\
\hline $\begin{array}{l}\text { Hydroxythiohomosildenafil } \\
\text { (Li et al., 2009b) } \\
\quad \mathrm{C}_{23} \mathrm{H}_{32} \mathrm{~N}_{6} \mathrm{O}_{4} \mathrm{~S}_{2}\end{array}$ & $\begin{array}{c}520 \\
521(M+H)\end{array}$ & & $\begin{array}{l}\left(\mathrm{CD}_{3} \mathrm{OD}\right): \text { : } \mathrm{H}-\mathrm{NMR} 0.99(\mathrm{t}, 3 \mathrm{H}), 1.54(\mathrm{t}, 3 \mathrm{H}), 1.82(\mathrm{~m}, 2 \mathrm{H}), 2.52(\mathrm{t}, \\
2 \mathrm{H}), 2.61(\mathrm{br} . \mathrm{t}, 4 \mathrm{H}), 2.89(\mathrm{t}, 2 \mathrm{H}), 3.07(\mathrm{br} . \mathrm{t}, 4 \mathrm{H}), 3.61(\mathrm{t}, 2 \mathrm{H}), 4.34(\mathrm{q}, \\
2 \mathrm{H}), 4.47(\mathrm{~s}, 3 \mathrm{H}), 7.39(\mathrm{~d}, \mathrm{~J}=8.4 \mathrm{~Hz}, 1 \mathrm{H}), 7.89(\mathrm{dd}, \mathrm{J}=8.4,2.4 \mathrm{~Hz}, 1 \mathrm{H}), \\
8.35(\mathrm{~d}, \mathrm{~J}=2.4 \mathrm{~Hz}, 1 \mathrm{H}) .\end{array}$ \\
\hline $\begin{array}{l}\text { Thiodimethylsildenafil } \\
\text { (Thiomethisosildenafil) } \\
\text { (Dimethylthiosildenafil) } \\
\text { (Sulfoaildenafil) } \\
\text { (Reepmeyer \& Avignon, 2009; Gratz } \\
\text { et al., 2009) } \\
\mathrm{C}_{23} \mathrm{H}_{32} \mathrm{~N}_{6} \mathrm{O}_{3} \mathrm{~S}_{2} \\
\end{array}$ & $\begin{array}{c}504 \\
505(M+H)\end{array}$ & & $\begin{array}{l}\left(\mathrm{CD}_{3} \mathrm{OD}\right):{ }^{1} \mathrm{H}-\mathrm{NMR} 0.99(\mathrm{t}, 3 \mathrm{H}), 1.04(\mathrm{~d}, 6 \mathrm{H}), 1.54(\mathrm{t}, 3 \mathrm{H}), 1.86(\mathrm{~m}, \\
4 \mathrm{H}), 2.87(\mathrm{~m}, 2 \mathrm{H}), 2.91(\mathrm{t}, 2 \mathrm{H}), 3.61(\mathrm{dd}, 2 \mathrm{H}), 4.34(\mathrm{q}, 2 \mathrm{H}), 4.49(\mathrm{~s}, \\
3 \mathrm{H}), 7.39(\mathrm{~d}, \mathrm{~J}=8.4 \mathrm{~Hz}, 1 \mathrm{H}), 7.89(\mathrm{dd}, \mathrm{J}=8.4,2.4 \mathrm{~Hz}, 1 \mathrm{H}), 8.33(\mathrm{~d}, \\
\mathrm{J}=2.4 \mathrm{~Hz}, 1 \mathrm{H}) .\end{array}$ \\
\hline 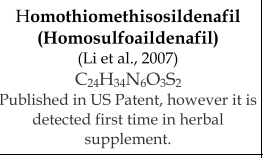 & $\begin{array}{c}518 \\
519(M+H)\end{array}$ & & $\begin{array}{l}\left(\mathrm{CDCl}_{3}\right): 1.01(\mathrm{t}, 3 \mathrm{H}), 1.04(\mathrm{~d}, 6 \mathrm{H}), 1.21(\mathrm{t}, 3 \mathrm{H}), 1.86(\mathrm{~m}, 4 \mathrm{H}), 2.11 \\
(\mathrm{~m}, 2 \mathrm{H}), 2.95(\mathrm{t}, 2 \mathrm{H}), 2.99(\mathrm{~m}, 2 \mathrm{H}), 3.66(\mathrm{dd}, 2 \mathrm{H}), 4.28(\mathrm{t}, 2 \mathrm{H}), 4.53(\mathrm{~s}, \\
3 \mathrm{H}), 7.19(\mathrm{~d}, \mathrm{~J}=8.4 \mathrm{~Hz}, 1 \mathrm{H}), 7.86(\mathrm{dd}, \mathrm{J}=8.4,2.4 \mathrm{~Hz}, 1 \mathrm{H}), 8.82(\mathrm{~d}, \\
\mathrm{J}=2.4 \mathrm{~Hz}, 1 \mathrm{H}) . \\
13 \mathrm{C}-\mathrm{NMR}\left(\mathrm{CDCl}_{3}\right): 11.03,14.2,19.5,22.4,22.58,27.8,39.6,50.5,52.4, \\
72.5,113.3,120.2,129.6,130.8,132.2,132.58,134.1,146.3,146.7, \\
159.76,172.06 .\end{array}$ \\
\hline $\begin{array}{c}\text { Dimethylsildenafil } \\
\text { (Aildenafil) (Methisosildenafil) } \\
\text { (Wang et al., 2007) } \\
\mathrm{C}_{23} \mathrm{H}_{32} \mathrm{~N}_{6} \mathrm{O}_{4} \mathrm{~S} \\
\end{array}$ & $\begin{array}{c}488 \\
489(M+H)\end{array}$ & & \begin{tabular}{|l|}
$\left(\mathrm{CDOD}_{3}\right): 0.99(\mathrm{t}, 3 \mathrm{H}), 1.04(\mathrm{~d}, 6 \mathrm{H}), 1.47(\mathrm{t}, 3 \mathrm{H}), 1.81(\mathrm{~m}, 2 \mathrm{H}), 1.89$ \\
$(\mathrm{t}, 2 \mathrm{H}), 2.87(\mathrm{~m}, 4 \mathrm{H}), 3.61(\mathrm{dd}, 2 \mathrm{H}), 4.23(\mathrm{~s}, 3 \mathrm{H}), 4.3(\mathrm{q}, 2 \mathrm{H}), 7.35(\mathrm{~d}$, \\
$\mathrm{J}=8.8 \mathrm{~Hz}, 1 \mathrm{H}), 7.87(\mathrm{dd}, \mathrm{J}=8.8,2.4 \mathrm{~Hz}, 1 \mathrm{H}), 8.17(\mathrm{~d}, \mathrm{~J}=2.4 \mathrm{~Hz}, 1 \mathrm{H})$. \\
\end{tabular} \\
\hline $\begin{array}{l}\text { Dimethylhomosildenafil* } \\
\text { (Homomethisosildenafil) } \\
\qquad \mathrm{C}_{24} \mathrm{H}_{34} \mathrm{~N}_{6} \mathrm{O}_{4} \mathrm{~S}\end{array}$ & $\begin{array}{c}502 \\
503(M+H)\end{array}$ & & $\begin{array}{l}\left(\mathrm{CDCl}_{3}\right): 1.01(\mathrm{t}, 3 \mathrm{H}), 1.05(\mathrm{~d}, 6 \mathrm{H}), 1.19(\mathrm{t}, 3 \mathrm{H}), 1.84(\mathrm{~m}, 2 \mathrm{H}), 1.93(\mathrm{t}, 2 \mathrm{H}) \\
2.05(\mathrm{~m}, 2 \mathrm{H}), 2.93(\mathrm{t}, 2 \mathrm{H}), 3.03(\mathrm{~m}, 2 \mathrm{H}), 3.68(\mathrm{dd}, 2 \mathrm{H}), 4.26(\mathrm{t}, 2 \mathrm{H}), 4.27 \\
(\mathrm{~s}, 3 \mathrm{H}), 7.16(\mathrm{~d}, \mathrm{~J}=8.8 \mathrm{~Hz}, 1 \mathrm{H}), 7.84(\mathrm{dd}, \mathrm{J}=8.8,2 \mathrm{~Hz}, 1 \mathrm{H}), 8.81(\mathrm{~d}, \mathrm{~J}=2 \mathrm{~Hz} \\
1 \mathrm{H}), 10.84(\text { br.s,1H) (in Table } 2)\end{array}$ \\
\hline $\begin{array}{c}\text { Nor-carbodenafil } \\
\text { Desmethylcarbodenafil } \\
\text { (Piazza \& Pamukçu, 2001) } \\
\mathrm{C}_{23} \mathrm{H}_{30} \mathrm{~N}_{6} \mathrm{O}_{3} \\
\text { Published in US Patent, however, it } \\
\text { is detected first time in herbal } \\
\text { supplement } \\
\end{array}$ & $\begin{array}{c}438 \\
439(M+H)\end{array}$ & & $\begin{array}{l}\left(\mathrm{CDCl}_{3}\right): 1.01(\mathrm{t}, 3 \mathrm{H}), 1.59(\mathrm{t}, 3 \mathrm{H}), 1.84(\mathrm{~m}, 2 \mathrm{H}), 2.34(\mathrm{~s}, 3 \mathrm{H}), 2.47 \\
(\mathrm{br} . \mathrm{H}, 4 \mathrm{H}), 2.9(\mathrm{t}, 2 \mathrm{H}), 3.56 \& 3.78(\mathrm{br} . \mathrm{s}, 4 \mathrm{H}), 4.25(\mathrm{~s}, 3 \mathrm{H}), 4.32(\mathrm{q}, 2 \mathrm{H}), \\
7.05(\mathrm{~d}, \mathrm{~J}=8.4 \mathrm{~Hz}, 1 \mathrm{H}), 7.55(\mathrm{dd}, \mathrm{J}=8.4,2.4 \mathrm{~Hz}, 1 \mathrm{H}), 8.55(\mathrm{~d}, \mathrm{~J}=2.4 \mathrm{~Hz}, \\
1 \mathrm{H}), 10.9(\mathrm{~s}, 1 \mathrm{H}) . \\
13 \mathrm{C}-\mathrm{NMR} 14.3,14.8,22.6,28.1,38.4,42.5(\text { br. s), 46.3, 48(br.s), } \\
55.3 \text { (br.s), 65.87, 113.2, 120.2, 124,7, 129.16, 130,7, 132.1, 138.7, 146.9, } \\
147.6,153.9,157.6,169.4 .\end{array}$ \\
\hline $\begin{array}{c}\text { Nor-acetildenafil } \\
\text { Demethylhongdenafil } \\
\text { (Reepmeyer \& Woodruff, 2007) } \\
\mathrm{C}_{24} \mathrm{H}_{32} \mathrm{~N}_{6} \mathrm{O}_{3}\end{array}$ & $\begin{array}{c}452 \\
453(M+H)\end{array}$ & & $\begin{array}{l}\left(\mathrm{CD}_{3} \mathrm{OD}\right): 0.99(\mathrm{t}, 3 \mathrm{H}), 1.46(\mathrm{t}, 3 \mathrm{H}), 1.8(\mathrm{~m}, 2 \mathrm{H}), 2.31(\mathrm{~s}, 3 \mathrm{H}), 2.57 \& \\
2.67(\mathrm{br} \mathrm{s}, 8 \mathrm{H}), 2.86(\mathrm{t}, 2 \mathrm{H}), 3.93(\mathrm{~s}, 2 \mathrm{H}), 4.21(\mathrm{~s}, 3 \mathrm{H}), 4.28(\mathrm{q}, 2 \mathrm{H}) \\
7.23(\mathrm{~d}, \mathrm{~J}=8.8 \mathrm{~Hz}, 1 \mathrm{H}), 8.16(\mathrm{dd}, \mathrm{J}=8.8,2.2 \mathrm{~Hz}, 1 \mathrm{H}), 8.46(\mathrm{~d}, \mathrm{~J}=2.2 \mathrm{~Hz}, \\
1 \mathrm{H})\end{array}$ \\
\hline $\begin{array}{l}\text { Dimethylacetildenafil* } \\
\text { (Goker et al., 2010) } \\
\quad \mathrm{C}_{25} \mathrm{H}_{34} \mathrm{~N}_{6} \mathrm{O}_{3}\end{array}$ & $\begin{array}{c}466 \\
467(M+H)\end{array}$ & & $\begin{array}{l}\left(\mathrm{CDCl}_{3}\right): 1.04(\mathrm{t}, 3 \mathrm{H}), 1.05(\mathrm{~d}, 6 \mathrm{H}), 1.63(\mathrm{t}, 3 \mathrm{H}), 1.83(\mathrm{t}, 2 \mathrm{H}), 1.89(\mathrm{~m}, \\
2 \mathrm{H}), 2.9(\mathrm{~m}, 2 \mathrm{H}), 2.93(\mathrm{t}, 2 \mathrm{H}), 3.02(\mathrm{~m}, 2 \mathrm{H}), 3.79(\mathrm{~s}, 2 \mathrm{H}), 4.28(\mathrm{~s}, 3 \mathrm{H}) \\
4.37(\mathrm{q}, 2 \mathrm{H}), 7.09(\mathrm{~d}, \mathrm{~J}=8.8 \mathrm{~Hz}, 1 \mathrm{H}), 8.16(\mathrm{dd}, \mathrm{J}=8.8,2 \mathrm{~Hz}, 1 \mathrm{H}), 9.15(\mathrm{~d}, \\
\mathrm{J}=2 \mathrm{~Hz}, 1 \mathrm{H}), 10.85(\mathrm{br} . \mathrm{s}, 1 \mathrm{H}) .\end{array}$ \\
\hline $\begin{array}{l}\text { Dapoxetine HCl } \\
\text { (Li et al., 2009b) } \\
\mathrm{C}_{21} \mathrm{H}_{23} \mathrm{NO} . \mathrm{HCl}\end{array}$ & $\begin{array}{c}305 \\
306(M+H)\end{array}$ & & $\begin{array}{l}\left(\mathrm{CDCl}_{3}\right): 2.2(\mathrm{~s}, 6 \mathrm{H}), 2.34-2.45(\mathrm{~m}, 1 \mathrm{H}), 2.59-2.7(\mathrm{~m}, 1 \mathrm{H}), 3.55-3.63 \\
(\mathrm{~m}, 1 \mathrm{H}), 3.86-3.9(\mathrm{~m}, 1 \mathrm{H}), 4.02-4.4(\mathrm{~m}, 1 \mathrm{H}), 6.62(\mathrm{~d}, \mathrm{~J}=7.6 \mathrm{~Hz}, 1 \mathrm{H}) \\
7.19-7.5(\mathrm{~m}, 9 \mathrm{H}), 7.74-7.77(\mathrm{~m}, 1 \mathrm{H}), 8.2-8.24(\mathrm{~m}, 1 \mathrm{H}) .\end{array}$ \\
\hline
\end{tabular}

*Detected in our lab. for the first time by us.

Table 1. Some synthetic PDE-5 inhibitors as analogues sildenafil and tadalafil were isolated by us. 


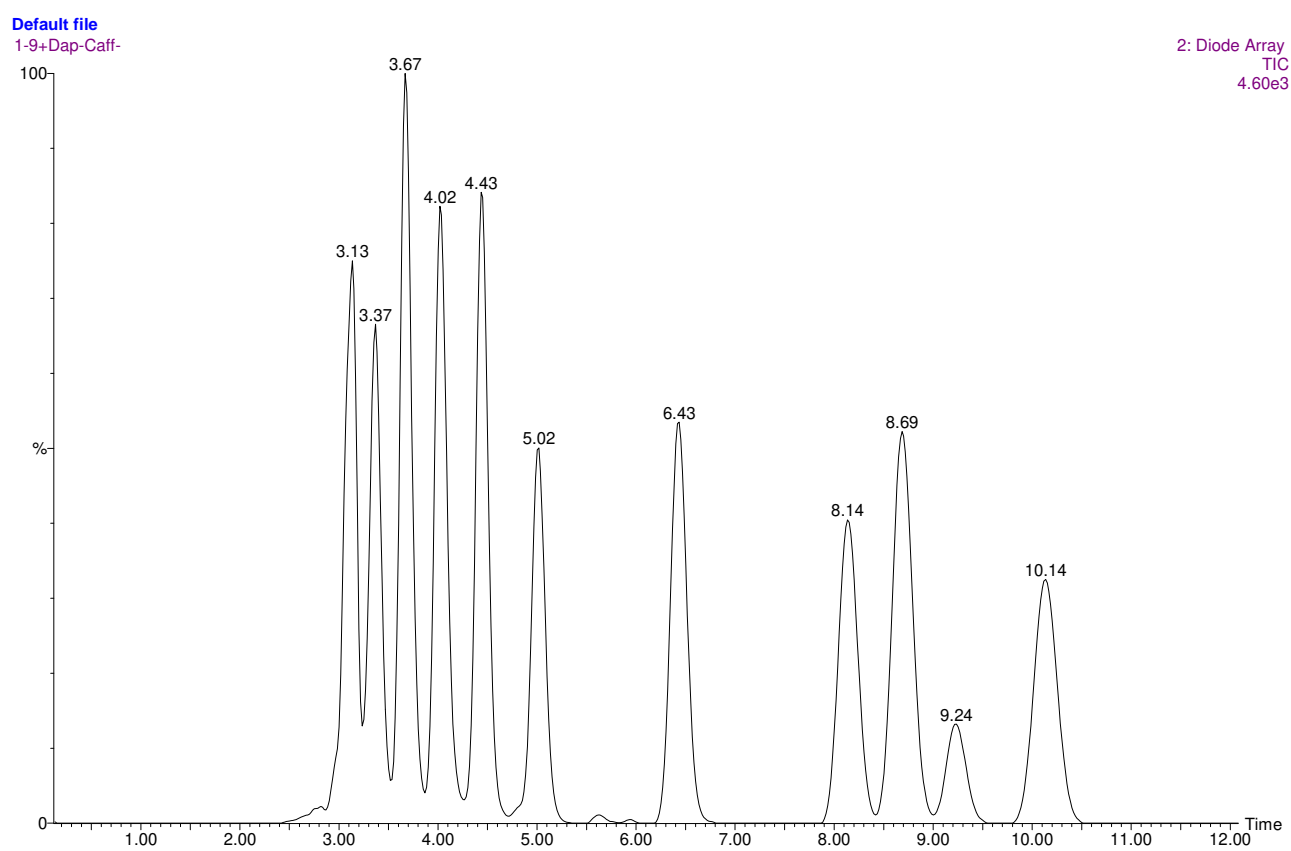

Fig. 9. HPLC chromotogram of 3.13 (Caffeine), 3.37 (Dimethylacetildenafil), 3.67 (Udenafil), 4.02 (Noracetildenafil), 4.43 (Dimethylsildenafil), 5.02 (Dimethylhomosildenafil), 6.43 (Hydroxythiohomosildenafil), 8.14 (Thiomethisosildenafil), 8.69 (Thiosildenafil), 9.24 (Dapoxetine $\mathrm{HCl}), 10.14$ (Thiohomomethisosildenafil) (RT : Retention times as $\mathrm{min}$ ).

Dapoxetin $\mathrm{HCl}$. Dapoxetine $\mathbf{H C l}$, is reported to be a selective serotonin reuptake inhibitor under investigation for the treatment of premature ejaculation (PE) (Li et al., 2009a). Since we have met this compound in one of the herbal drinks for adults, we have added it to our list. As it is well known, caffeine is very common for this kind of health supplements, it was added to chromatogram as well.

\subsection{Natural aphrodisiacs}

Formulas, ${ }^{1} \mathrm{H}-13 \mathrm{C}-\mathrm{NMR}$ spectra, $\mathrm{ESI}(+) \mathrm{m} / \mathrm{e}$ values and LC-MS ion chromatograms of natural aphrodisiacs are given in Table 2 and Fig. 10.

L- Arginine is not herb, but a nonessential amino acid, it is found naturally in foods such as meat, dairy, poultry and fish, it also may be synthesized in the laboratory, in spite of there is insufficient evidence to rate effectiveness for male fertility and female sexual problem, but it is possible to see the ingredients of many aphrodisiacs, recently. It is also available as oral Larginine supplements, which some product manufacturers market as a "natural Viagra" (Stanislavov \& Nikolova, 2003). 


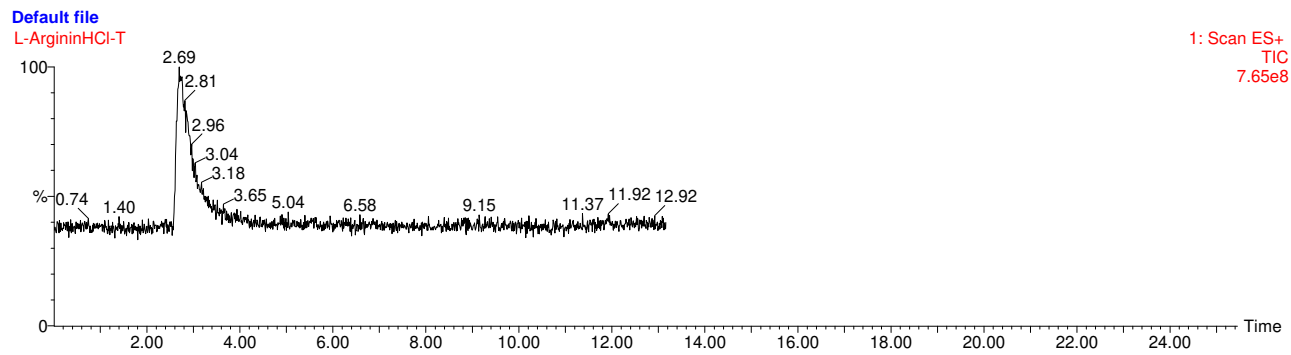

Default file

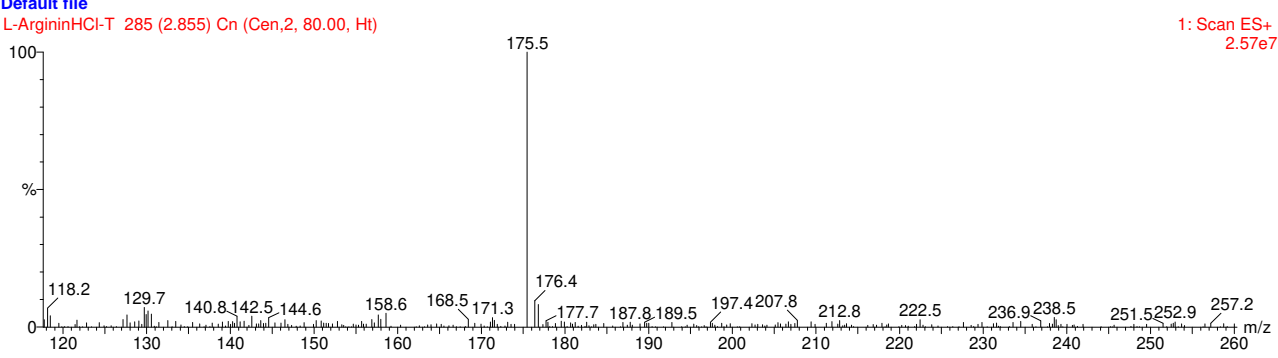

Default file

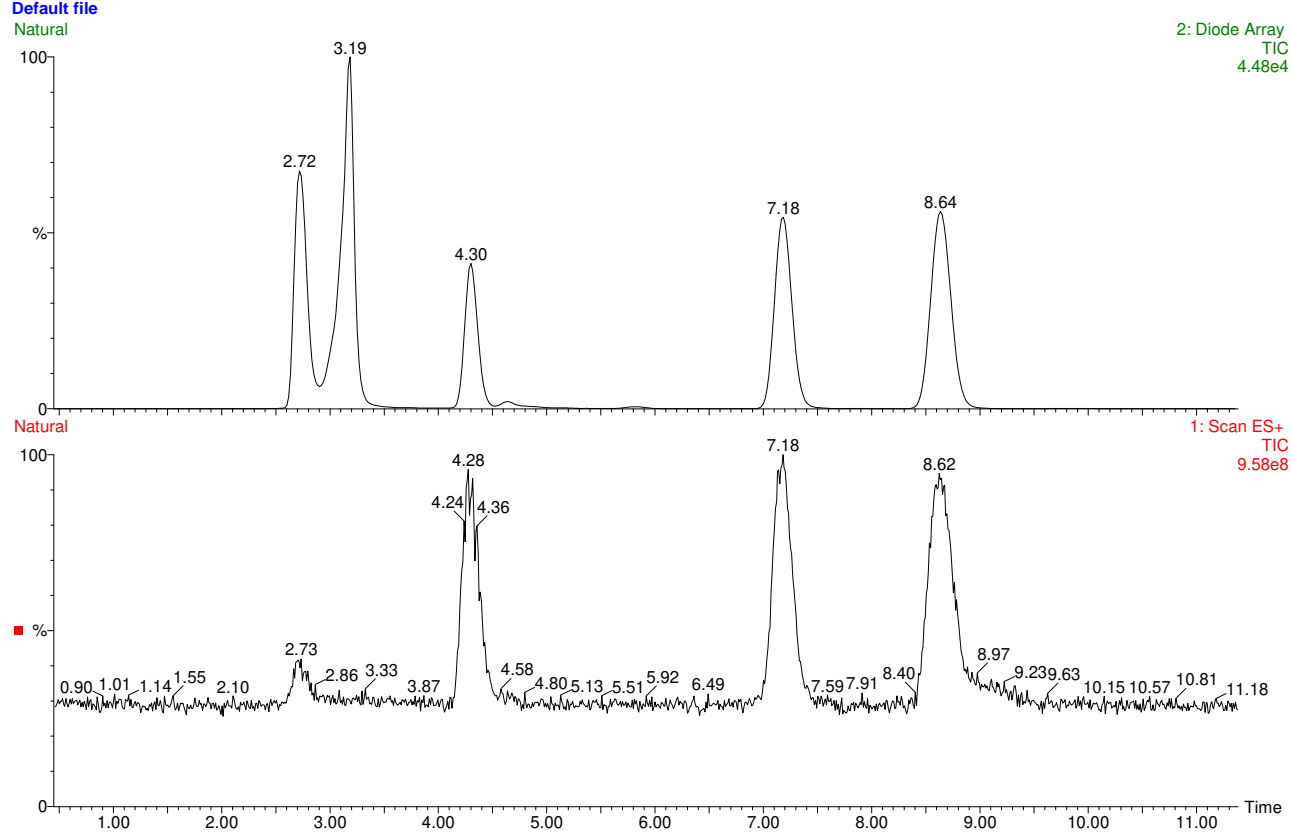

Fig. 10. HPLC-MS ion chromatogram of 2.69 (L-Arginin $\mathrm{HCl}$ ), 2.72 (Inosine), 3.19 (Icariin), 4.3 (Yohimbin), 7.18 (Imperatonin), 8.64 (Osthole). (RT : Retention times as min). 


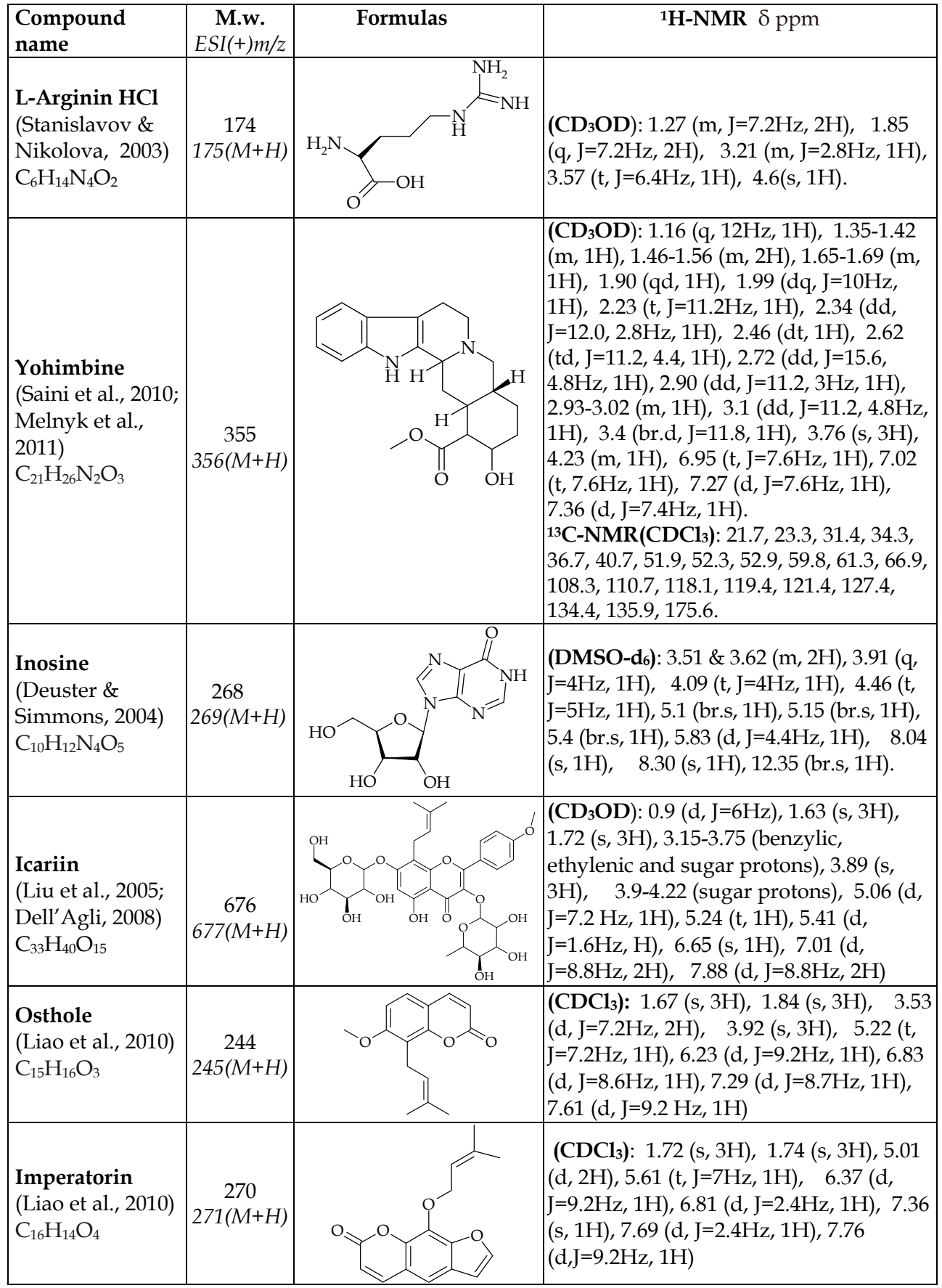

Table 2. Natural aphrodisiacs were isolated by us from some herbal supplements. 
Yohimbine is an alkaloid which are found naturally in Pausinystalia yohimbe, a classical aphrodisiac which has been recently revalued for its pro-sexual properties and extensively commercialized without control in some countries (Saini et al., 2010, Melnyk et al., 2011) . However, there is little evidence on its efficacy in the treatment of ED and it is, therefore, not currently recommended (Albersen et al., 2010).

Inosine is a nucleoside consisting of ribose and hypoxanthine, most commonly found in supplements that claim to be "energy promoters", naturally it is found in brewer's yeast and organ meats, however it can be synthesized in laboratory (Deuster \& Simmons, 2004). Their nerve-stimulating action probably enhances sexual functions or help to remedy sexual functions when these are reduced due to degeneration of nerve tissue.

Icariin is one of the primary active component of Epimedium extracts, which has been used to treat impotence and improve sexual function by acting as PDE5 inhibitor as nafil derivatives (Liu et al., 2005; Dell'Agli, 2008). Since the illegally using of synthetic nafil derivatives have been prohibited, recently icariin has become very attractive by the drug manufacturers and suppliers, because of its natural character. Nowadays, the percentage ratio of Icariin in the commercial Epimedium extracts has been increased over 80-90. However there is no sufficient information about its pharmacological profiles and safety. Further investigation needs to be done to examine any benefits that could occur from supplements.

Osthole and imperatorin, coumarin compounds have been reported to exhibit various biological activities (Liao et al., 2010). It was reported that, both of them were found to help relax the corpus cavernosa of the penis, which would potentially help with blood flow, in phenylephrine-precontracted endothelium-intact rabbit corpus cavernosum (Chen et al., 2000; Chiou et al., 2001), however there is no information for human uses.

Herbal medications are being progressively utilized all over the world and it is believed that herbal remedies are not hazards, however some adverse reactions have been increased. Tribulus terrestris is frequently used because of its aphrodisiac effect. But there is an article (Talasaz, 2010), which reports a case of T-terrestris-induced hepatotoxicity, nephrotoxicity and neurotoxicity in an Iranian male patient.

In this text, we also present a new sildenafil analogue was found to have been added illegally to a herbal drinks marketed for the enhancement of sexual function. This analogue has never been found. Therefore, it prompted us to elucidate its structure. The structure was determined as 5-[5-[[(3,5-dimethyl-1-piperazinyl]sulfonyl]-2-propoxyphenyl]-1,6-dihydro-1methyl-3-propyl-7H-pyrazolo [4,3-d]pyrimidin-7-one. Owing to the inclusion of a methylene group in dimethylsildenafil, the detected compound was called Dimethyhomosildenafil. The sample was purified with column chromatography. The IR, HPLC-/MS (ESI+), and completely assigned NMR data of dimethylhomosildenafil have been observed.

\section{Material and methods}

\subsection{Equipments}

Uncorrected melting points were measured on an Büchi B-540 capillary melting point apparatus. ${ }^{1} \mathrm{H}(400 \mathrm{MHz})$ and ${ }^{13} \mathrm{C}(100 \mathrm{MHz}) \mathrm{NMR}$ spectra were recorded employing a 
VARIAN MERCURY $400 \mathrm{MHz}$ FT spectrometer, with $\mathrm{CDCl}_{3}$ as solvent. Chemical shifts $(\delta)$ are in ppm relative to TMS. The LC/MS were taken on a Waters Micromass ZQ connected with Waters Alliance HPLC, using ESI(+) method, with C-18 column. Elemental analyses were performed by Leco CHNS-932. The infrared spectrum was recorded in the 600-3600 $\mathrm{cm}^{-1}$ range using a Jasco FT-IR-420 spectrometer and KBr pellets.

\subsection{Extraction and isolation}

The water contents of the alüminyum can $(250 \mathrm{ml})$ were extracted with the mixture of dichloromethane-methanol (95:5) and evaporated, residue was directly carried out to a open column with silica gel $60(0.04-0.063 \mathrm{~mm})$ and eluted with dichloromethane-isopropanol (97:3). Fractions were collected and analyzed by TLC. All of the fractions having the target compound were collected and the solvent was evaporated and crystallization of the residue from ethanol gave $0.021 \mathrm{~g}$ of white powder compound was obtained, m.p: $189-190^{\circ} \mathrm{C}$, Anal. Calcd. for $\mathrm{C}_{24} \mathrm{H}_{34} \mathrm{~N}_{6} \mathrm{O}_{4}$ S. $0.5 \mathrm{HOH}$ : C 56.34, H 6.90, N 16.42, S 6.27. Found: C 56.47, H 6.95, N 16.21, S 6.28.

\subsection{Structure identification}

\subsubsection{NMR correlation data of dimethylhomosildenafil}

Dimethylhomosildenafil was dissolved in $\mathrm{CDCl}_{3}$ and subjected to $1 \mathrm{D}$ and $2 \mathrm{D} \mathrm{NMR}$ spectroscopic analysis $\left({ }^{1} \mathrm{H},{ }^{13} \mathrm{C}, \mathrm{DEPT}\right.$, homo-COSY, HSQC and HMBC). The data are shown in Table 2.

\subsubsection{Analysis condition of HPLC/MS}

LC-MS coupled with positive and negative (ESI+) Electro Spray method was used to determine its molecular weight. The HPLC of LC/MS was carried out on a column XTerra ${ }^{\circledR}$ MS C-18 $(4.6$ X250 mm,5 $\mu \mathrm{m})$ with Acetonitrile: Methanol:0.05 M Ammonium acetate in water (55:20:25) as mobile phase. The flow rate was $0.9 \mathrm{~mL} / \mathrm{min}$, the injection volume was $5 \mu \mathrm{L}$ and the appropriate running time (at least $15 \mathrm{~min}$ ). The eluate was monitored by a photo-diode array detector at $254 \mathrm{~nm}$. The analytical condition of mass was as follows: capillary voltage $: 3.41 \mathrm{kV}$, cone voltage : $26 \mathrm{~V}$, source temperature : $100{ }^{\circ} \mathrm{C}$ : desolvation temperature : $350^{\circ} \mathrm{C}$. The HPLC chromatogram of dimethylhomosildenafil is given in Fig 2 with 5.02 min. $r_{t}$ values. This method was carried out all the given HPLCMS analysis in this text.

Table 3 shows the ${ }^{1} \mathrm{H}-\mathrm{NMR},{ }^{13} \mathrm{C}-\mathrm{NMR}, \mathrm{DEPT}$, COSY, HSQC and HMBC spectral data of isolated compound 1, which were similar to that of dimethylsildenafil. The spectroscopic numbering used is given in Table 3. The difference of this compound, than dimethylsildenafil is related with ether protons connected to the C-19. Here is one more metyhlene group as propoxy. The 3,5-dimethyl protons of piperazine were observed at $\delta \mathrm{H}$ $1.05(\mathrm{~d}, 6 \mathrm{H})$ as expected, in the HMBC spectrum the correlation of $\mathrm{H}-28,29 / \mathrm{H}-24,26$, DEPT and HSQC results indicated that dimethyl group attached to C24-26. Since the 2,6diequatorial methyl groups would be lower energetic form, so the configuration is established as a cis diequatorial methyl configuration as shown in Table 1 as it is in the 
methisosildenafil (Reepmeyer \& Avignon, 2009). The IR spectrum of dimethylhomosildenafil is given in Fig 11. This compound also must be put on the inspection list for illegal health-related substances because of the unknown safety and toxicity profile.

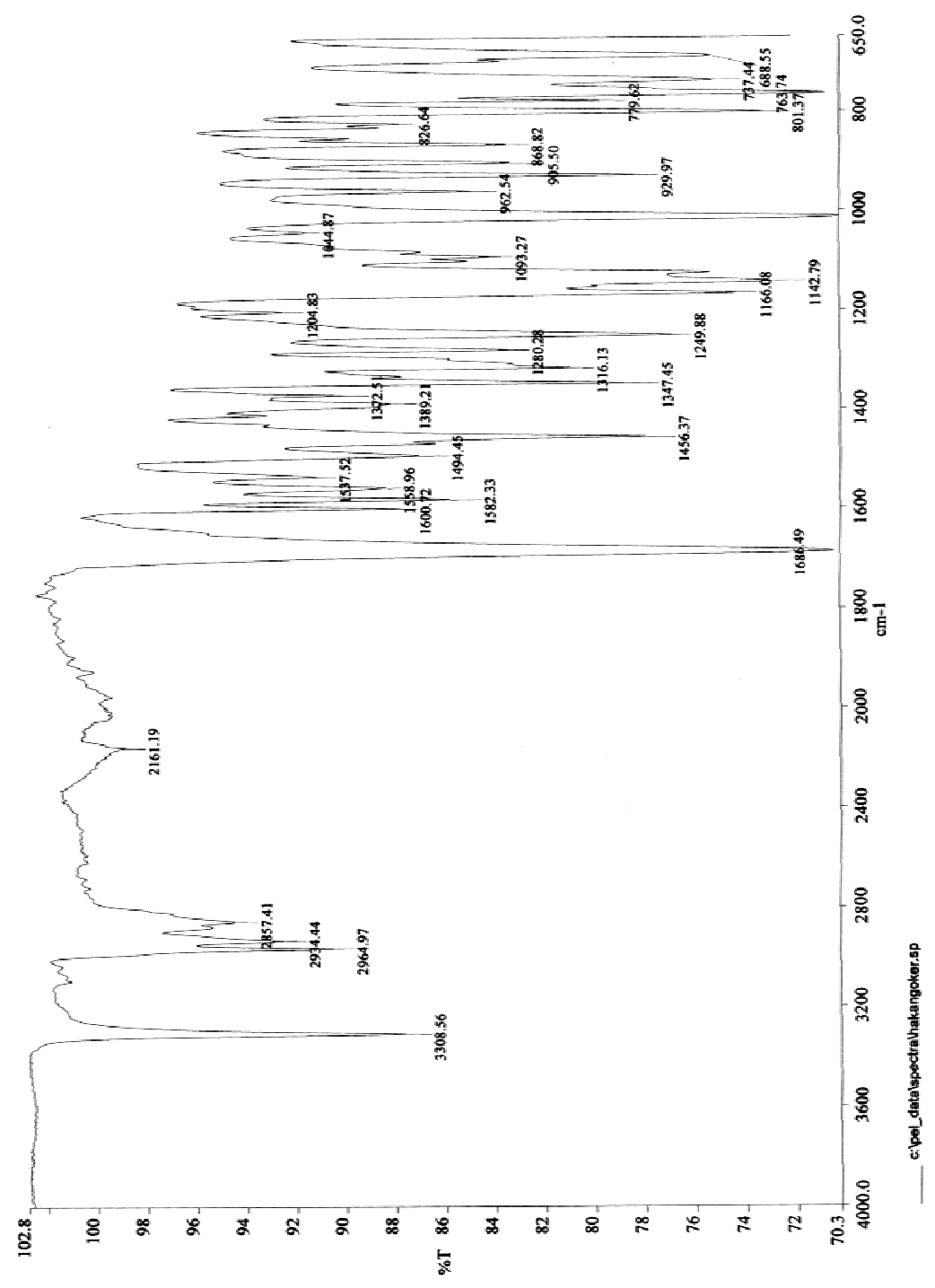

Fig. 11. IR spectrum of Dimethylhomosildenafil. 


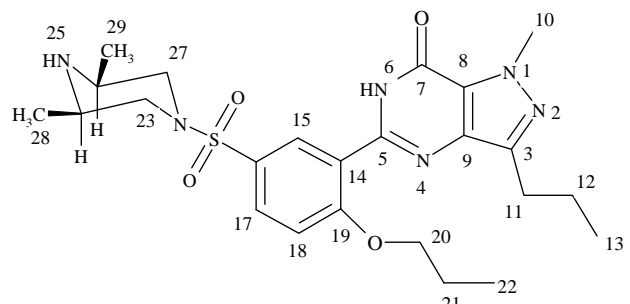

\begin{tabular}{|c|c|c|c|c|c|}
\hline No & ${ }^{13} \mathrm{C}$ & $1 \mathbf{H}$ & DEPTa & COSY & HMBC \\
\hline 3 & 146.7 & -- & & & C-3/H-12 C-3/H-11 \\
\hline 5 & 147.2 & --- & & & C-5/H-15 \\
\hline 6 & -- & 10.84(br.s,1H) & & & -- \\
\hline 7 & 153.8 & -- & & & -- \\
\hline 8 & 124.7 & --- & & & C-8/H-10 \\
\hline 9 & 138.6 & -- & & & $\mathrm{C}-9 / \mathrm{H}-11$ \\
\hline 10 & 38.4 & $4.27(\mathrm{~s}, 3 \mathrm{H})$ & 3 & & -- \\
\hline 11 & 27.99 & $2.93(\mathrm{t}, 2 \mathrm{H}, \mathrm{J}=7.2 \mathrm{~Hz})$ & 2 & H-11/H-12 & C-11/H-13, C-11/H-12 \\
\hline 12 & 22.5 & $1.84(\mathrm{~m}, 2 \mathrm{H}, \mathrm{J}=7.6 \mathrm{~Hz})$ & 2 & $\begin{array}{l}\mathrm{H}-12 / \mathrm{H}-11, \\
\mathrm{H}-12 / \mathrm{H}-13\end{array}$ & C-12/H-13, C-12/H-11 \\
\hline 13 & 14.3 & $1.01(\mathrm{t}, 3 \mathrm{H}, \mathrm{J}=7.6 \mathrm{~Hz})$ & 3 & $\mathrm{H}-13 / \mathrm{H}-12$ & C-13/H-12, C-13/H-11 \\
\hline 14 & 129.4 & -- & & -- & C-14/H-18 \\
\hline 15 & 131.1 & $8.81\left(\mathrm{~d}, 1 \mathrm{H}, J_{m}=2 \mathrm{~Hz}\right)$ & 1 & $\mathrm{H}-15 / \mathrm{H}-17$ & $\mathrm{C}-15 / \mathrm{H}-17$ \\
\hline 16 & 121.2 & -- & & & C-16/H-18 \\
\hline 17 & 131.8 & $\begin{array}{c}\text { 7.84(dd, } 1 \mathrm{H}, J_{o}=8.8 \mathrm{~Hz} \\
\left.J_{m}=2 \mathrm{~Hz}\right)\end{array}$ & 1 & $\begin{array}{l}\mathrm{H}-17 / \mathrm{H}-18, \\
\mathrm{H}-17 / \mathrm{H}-15\end{array}$ & C-17/H-15 \\
\hline 18 & 113.2 & $7.16\left(\mathrm{~d}, 1 \mathrm{H}, J_{o}=8.8 \mathrm{~Hz}\right)$ & 1 & $\mathrm{H}-18 / \mathrm{H}-17$ & ---- \\
\hline 19 & 159.7 & -- & & & $\begin{array}{l}\text { C-19/H-15, C-19/H-17 } \\
\text { C-19/H-18, C-19/H-20 }\end{array}$ \\
\hline 20 & 72.1 & $4.26(\mathrm{t}, 2 \mathrm{H}, \mathrm{J}=7.2 \mathrm{~Hz})$ & 2 & H-20/H-21 & $\mathrm{C}-20 / \mathrm{H}-21, \mathrm{C} 20 / \mathrm{H}-22$ \\
\hline 21 & 22.54 & $2.05(\mathrm{~m}, 2 \mathrm{H}, J=6.8 \mathrm{~Hz})$ & 2 & $\begin{array}{l}\mathrm{H}-21 / \mathrm{H}-20, \\
\mathrm{H}-21 / \mathrm{H}-22 \\
\end{array}$ & C-21/H-20, C-21/H-22 \\
\hline 22 & 10.85 & $1.19(\mathrm{t}, 3 \mathrm{H}, \mathrm{J}=7.6 \mathrm{~Hz})$ & 3 & H-22/H-21 & $\mathrm{C}-22 / \mathrm{H}-21, \mathrm{C}-22 / \mathrm{H}-20$ \\
\hline 23,27 & 52.3 & $\begin{array}{c}1.93(\mathrm{t}, 2 \mathrm{H}, J=10.4 \mathrm{~Hz}, \\
\text { axial }) \& 3.68(\mathrm{dd}, 2 \mathrm{H}, \\
J=10.4 \mathrm{~Hz}, J=1.6 \mathrm{~Hz} \\
\text { equatorial })\end{array}$ & 2 & $\begin{array}{l}\text { H-23,H-27 } \\
\quad \text { axial/ } \\
\mathrm{H}-24, \mathrm{H}-26\end{array}$ & C-23,27/H-28,29 \\
\hline 24,26 & 50.5 & $\begin{array}{c}3.03(\mathrm{~m}, 2 \mathrm{H}, \mathrm{J}=3.6 \mathrm{~Hz} \\
\text { axial })\end{array}$ & 1 & $\begin{array}{c}\text { H-24,H-26/ } \\
\text { H-23,H-27 } \\
\text { axial } \\
\text { H-24,H-26/ } \\
\text { H-28,H-29 } \\
\end{array}$ & $\begin{array}{l}\text { C-24,26/H-28,29 } \\
\text { C-24,26/H-23,27 }\end{array}$ \\
\hline 28,29 & 19.5 & $1.05(\mathrm{~d}, 6 \mathrm{H}, \mathrm{J}=6.8 \mathrm{~Hz})$ & 3 & $\begin{array}{c}\mathrm{H}-28, \mathrm{H}-29 / \\
\mathrm{H}-24, \mathrm{H}-26\end{array}$ & --- \\
\hline
\end{tabular}

$\delta$ ppm in $\mathrm{CDCl}_{3}, J$ in $\mathrm{Hz}$ a) Number in DEPT is the number of attached protons.

Table 3. NMR data of Dimethylhomosildenafil. 


\section{Conclusion}

Oral PDE5 inhibitors are the treatment of choice for MED. The physiological mechanism of erection involves release of nitric oxide (NO) in the corpus cavernosum as a result of sexual stimulation. NO then activates the enzyme guanylate cyclase, which results in increased levels of cyclic guanosine monophosphate (cGMP), leading to smooth muscle relaxation in blood vessels supplying the corpus cavernosum and allowing inflow of blood. Nafil derivatives have no direct relaxant effect on isolated human corpus cavernosum, but enhance the effect of NO by inhibiting PDE5, which is responsible for degredation of cGMP in the corpus cavernosum. When sexual stimulation causes local release of NO, inhibition of PDE5 by nafil analogues causes increased levels of cGMP in the corpus cavernosum, resulting in smooth muscle relaxation and inflow of blood to the corpus cavernosum. This mode of action means that PDE5 inhibitors are ineffective without sexual stimulation. The PDE-5 inhibitors have helped many men with MED, and the FDA indicate that Sildenafil citrate, Vardenafil $\mathrm{HCl}$ and Tadalafil are safe and well-tolerated when taken as directed by men who have gotten approval from their doctors. These drugs must not been used without medical examination or prescription. PDE- 5 inhibitors also increase the risk of a variety of cardiovascular diseases, including heart attack, myocardial infarction, and sudden death. The medication may interact with other drugs which should be mortal, e.g. synergic effect with alpha-blockers. Other side effects associated with PDE-5 drugs, such as priapism, severe hypotension, increased intraocular pressure and sudden hearing loss and blidness. The PDE-5 inhibitors must not buy over the internet or other non-standart source, otherwise, the men run several risk, such as it should be counterfeit product that does not have the legal structural compound which has been untested for safety or no same purity as the real drug.

\section{Acknowledgment}

We thank Prof. Dr. Erden Banoğlu (Gazi University, Faculty of Pharmacy, Ankara) for providing sample of Homothiomethisosildenafil which was also isolated from the herbal dietary supplement by him. Central Instrumental Analysis Lab. of Pharmacy, Faculty of Ankara University provided support for acquisition of the IR, NMR, HPLC-MS spectrometers and elemental analyzer used in this work.

\section{References}

Albersen, M.; Shindel, A. W.; Mwamukonda, K. B. \& Lue, T. F. (2010). The future is today: emerging drugs for the treatment of erectile dysfunction, Expert Opin. Emerging Drugs 15:467-480.

Bell, A. S. \& Palmer, M. J. (2011). Novel phosphodiesterase type 5 modulators: a patent survey (2008-2010), Expert Opinion on Therapeutic Patents 21:1631-1641.

Chen, J.; Chiou, W. F.; Chen, C. C. \& Chen, C. F. (2000). Effect of the plant-extract osthole on the relaxation of rabbit corpus cavernosum tissue in vitro. J. Urol. 163:1975-80.

Chiou, W. F.; Huang, Y. L.; Chen, C. F. \& Chen, C. C. (2001). Vasorelaxing Effect of Coumarins from Cnidium monnieri on Rabbit Corpus Cavernosum. Planta Med. 67:282-284. 
Dell'Agli, M. D.; Galli, G. V.; Cero, E. D.; Belluti, F.; Matera, R. \& Zironi, E. (2008). Potent inhibition of human phosphodiesterase-5 by Icariin Derivatives, J. Nat. Prod. 71: 15131517.

Deuster, P. A. \& Simmons, R. G. (2004) Dietary Supplements and Military Divers. A Synopsis for Undersea Medical Officers, Available from: www.usuhs.mil/mem/ hpl/DietarySupplementUMO.pdf.

Ge, X.; Li, L.; Koh, H. L. \& Low, M.Y. (2011). Identification of a new sildenafil analogue in a health supplement, J. Pharm. Biomed. Anal. 56:491-496.

Göker, H.; Coskun, M. \& Alp, M. (2010). Isolation and identification of a new acetildenafil analogue used to adulterate a dietary supplement: dimethylacetildenafil, Turk J Chem. 157-163.

Gratz, S. R.; Zeller, M.; Mincey, D. W. \& Flurer, C. L. (2009). Structural characterization of sulfoaildenafil, an analog of sildenafil, J. Pharm. Biomed. Anal. 50:228-231.

Häberli, A.; Girard, P.; Low, M. Y. \& Ge, X. (2010). Isolation and structure elucidation of an interaction product of aminotadalafil found in an illegal health food product, $J$. Pharm. Biomed. Anal. 53:24-28.

Hasegawa, T.; Takahashi, K.; Saijo, M.; Ishii, T.; Nagata, T.; Kurihara, M.; Haishima, Y.; Goda, Y. \& Kawahara, N. (2009). Isolation and Structural Elucidation of Cyclopentynafil and N-Octylnortadalafil Found in a Dietary Supplement, Chem Pharm. Bull. 57:185-189.

Jung, J. Y.; Kim, S. K.; Kim, B. S.; Lee, S. H.; Park, Y. S.; Kim, S. J.; Choi, C.; Yoon, S. I.; Kim, J. S.; Cho, S. D.; Im, G. J.; Lee, S. M.; Jung, J. W. \& Lee, Y. S. (2008). The penile erection efficacy of a new phosphodiesterase type 5 inhibitor, mirodenafil (SK3530), in rabbits with acute spinal cord injury. J. Vet. Med. Sci., 70:1199-204.

Keating, G. M. \& Scott, L. J. (2003). Vardenafil: a review of its use in erectile dysfunction, Drugs, 63:2673-703.

Kesting, J. R.; Huang, J. \& Sørensen, D. (2010). Identification of adulterants in a Chinese herbal medicine by LC-HRMS and LC-MS-SPE/NMR and comparative in vivo study standarts in a hypertensive rat model, J. Pharm. Biomed. Anal. 51:705-711.

Kurita, H.; Mizuno, K.; Kuromi, K.; Suzuki, N.; Ueno, C.; Kamimura, M.; Fujiwara, A.; Owada, K.; Ogo, N. \& Yamamoto, M. (2008). Identification of Aminotadalafil and its Stereoisomers Contained in Health Foods Using Chiral Liquid ChromatographyMass Spectrometry, J. Health Science, 54:310-314.

Lai, K. C.; Liu, Y. C.; Tseng, M. C.; Lin, Y. L. \& Lin, J. H. (2007a). Isolation and Identification of a Vardenafil Analogue in a Dietary Supplement, J. Food Drug Anal. 15:220-227.

Lai, K. C.; Liu, Y. C.; Tseng, M. C.; Lin, Y. L. \& Lin, J. H. (2007b). Isolation and Identification of a Vardenafil Analogue in a Functional Food Marketed for Penile Erectile Dysfunction, J. Food Drug Anal. 15:133-138.

Lam, Y. H.; Poon, W. T.; Lai, C. K.; Chan, A. Y. W. \& Mak, T. W. L. (2008). Identification of a novel vardenafil analogue in herbal product, J. Pharm. Biomed. Anal. 46:804-807.

Langtry, H. D. \& Markham, A. (1999). Sildenafil: a review of its use in erectile dysfunction, Drugs, 57:967-89.

Lee, H. M.; Kim, S. C.; Jang, Y. M.; Kwon, S. W. \& Lee, B. J. (2011). Separation and structural elucidation of a novel analogue of vardenafil included as an adulterant in a dietary supplement by liquid chromatography-electrospray ionization mass spectrometry, infrared spectroscopy and nuclear magnetic resonance spectroscopy, J. Pharm. Biomed. Anal. 54:491-496. 
Li, L.; Low, M. Y.; Ge, X.; Bloodworth, B. C. \& Koh, H. L. (2009a). Isolation and structural elucidation of dapoxetine as an adulterant in a health supplement used for sexual performance enhancement, J. Pharm. Biomed. Anal. 50:724-728.

Li, L.; Low, M. Y.; Aliwarga, F.; Teo, J.; Ge, X. W.; Zeng, Y.; Bloodworth, B. C. \& Koh, H. L. (2009b). Isolation and identification of hydroxythiohomosildenafil in herbal dietary supplements sold as sexual performance enhancement products, Food Addit. Contam. 26:145-151.

Li, S.; Ren, J.; Zhao, Y.; Lv, Q. \& Guo, J. (2007). Pyrazolopyrimidinethione Derivatives, Salts and Solvates, thereof, preparation methods and use thereof, US 2007/0219220.

Liao, P. C.; Chien, S. C.; Ho, C. L.; Wang, E. I. C.; Lee, S. C.; Kuo, Y. H.; Jeyashoke, N.; Chen, J.; Dong, W. C.; Chao, L. K. \& Hua, K. F. (2010). Osthole Regulates Inflammatory Mediator Expression through Modulating NF-kB, Mitogen-Activated Protein Kinases, Protein Kinase C, and Reactive Oxygen Species, J. Agric. Food Chem, 58: 10445-10451.

Lin, M. C.; Liu, Y. C.; Lin, Y. L. \& Lin, J. H. (2008). Isolation and Identification of a Novel Sildenafil Analogue Adulterated in Dietary Supplements, J Food Drug Anal. 16:15-20.

Lin, M. C.; Liu, Y. C.; Lin, Y. L. \& Lin, J. H. (2009). Identification of a Tadalafil Analogue Adulterated in a dietary Supplement, J. Food Drug Anal. 17:451-458.

Liu, R.; Li, A.; Sun, A.; Cui, J. \& Kong, L. (2005). Preparative isolation and purification of three flavonoids from the Chinese medicinal plant Epimedium koreamum Nakai by highspeed counter-current chromatography, J Chromatog. A, 1064:53-57.

Melnyk, J. P. \& Marcone, M. F. (2011). Aphrodisiacs from plant and animal sources-A review of current scienfitic literature, Food Research International. 44:840-850.

Meuleman, E. J. (2003). Review of tadalafil in the treatment of erectile dysfunction, Expert Opin. Pharmacother, 4:2049-56.

Park, H. J.; Jeong, H. K.; Chang, M. I.; Im, M. H.; Jeong, J. Y.; Choi, D. M.; Park, K.; Hong, M. K.; Youm, J.; Han, S. B; Kim, D. J.; Park, J. H. \& Kwon, S. W. (2007). Structure determination of new analogues of vardenafil and sildenafil in dietary supplements, Food Addit. Contam. 24:122-129.

Piazza, G. A. \& Pamukçu, R. (2001). Method of treating a patient having precancerous lesions with phenyl purinone derivatives, US 6,200,980.

Poon, W. T.; Lam, Y. H.; Lai, C. K.; Chan, A. Y. W. \& Mak, T. W. L. (2007). Analogues of erectile dysfunction drugs: an under-recognised threat, Hong Kong Med J. 13:359-63.

Reepmeyer, J. C. \& Woodruff, J. T. (2007). Use of liquid chromatography-mass spectrometry and a chemical cleavage reaction for the structure elucidation of a new sildenafil analogue detected as an adulterant in an herbal dietary supplement, J. Pharm. Biomed. Anal. 44:887-893.

Reepmeyer, J. C. \& D'Avignon, D. A. (2009). Structure elucidation of thioketone analogues of sildenafil detected as adulterants in herbal aphrodisiacs, J. Pharm. Biomed. Anal. 49:145-150.

Saini, N. K.; Singhal, M.; Srivastava, B. \& Sharma, S. (2010). Natural Plants Effective in Treatmen of Sexual Dysfunction: A Review, The Pharma Research, 4:206-224.

Salem, E. A.; Kendirci, M. \& Hellstrom, W. J. (2006). Udenafil, a long acting PDE5 inhibitor for erectile dysfunction Curr Opin. Investig. Drugs, 7:661-9.

Stanislavov, R. \& Nikolova, V. (2003) Treatment of Erectile Dysfunction with Pycnogenol and L-Arginine, J Sex Marital Therapy, 29:207-213. 
Talasaz, A. H.; Abbasi, M. R.; Abkhiz, S. \& Dahti-Khavidaki, S. (2010). Tribulus terrestrisinduced severe nephrotoxicity in a young healthy male, Nephrol Dial. Transplant. 25: 3792-3793.

Toque, H. A.; Teixeira, C. E.; Lorenzetti, R.; Okuyama, C. E.; Antunes, E. \& De Nucci, G. (2008). Pharmacological characterization of a novel phosphodiesterase type 5 (PDE5) inhibitor lodenafil carbonate on human and rabbit corpus cavernosum, European Journal of Pharmacology, 591:189-195.

Venhuis, B. J.; Zomer, G.; Hamzink, M.; Meiring, H. D.; Aubin, Y. \& Kaste, D. (2011). The identification of a nitrosated prodrug of the PDE-5 inhibitor aildenafil in a dietary supplement: A Viagra with a pop, J. Pharm. Biomed. Anal. 54:735-741.

Wang, J.; Jiang, Y.; Wang, Y.; Zhao, X.; Cui, Y. \& Gu, J. (2007). Liquid chromatography tandem mass spectrometry assay to determine the pharmacokinetics of aildenafil in human plasma, J. Pharm. Biomed. Anal. 44:231-235.

Wollein, U.; Eisenreich, W. \& Schramek, N. (2011). Identification of novel sildenafil-analogues in an adulterated herbal food supplement, J. Pharm. Biomed. Anal. 56:705-712.

Zou, P.; Hou, P.; Low, M. Y. \& Koh, H. L. (2006). Structural elucidation of a tadalafil analogue found as an adulterant of a herbal product, Food Addit. Contam. 23:446-451.

Zou, P.; Hou, P.; Oh, S. S. Y.; Chong, Y. M.; Bloodworth, B. C.; Low, M. Y. \& Koh, H. L. (2008). Isolation and identification of thiohomosildenafil and thiosildenafil in health supplements, J. Pharm. Biomed. Anal. 47:279-284. 


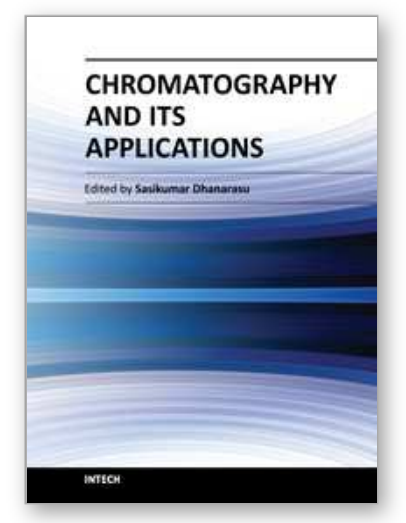

\author{
Chromatography and Its Applications \\ Edited by Dr. Sasikumar Dhanarasu
}

ISBN 978-953-51-0357-8

Hard cover, 224 pages

Publisher InTech

Published online 16, March, 2012

Published in print edition March, 2012

Chromatography is a powerful separation tool that is used in all branches of science, and is often the only means of separating components from complex mixtures. The Russian botanist Mikhail Tswett coined the term chromatography in 1906. The first analytical use of chromatography was described by James and Martin in 1952 , for the use of gas chromatography for the analysis of fatty acid mixtures. A wide range of chromatographic procedures makes use of differences in size, binding affinities, charge, and other properties. Many types of chromatography have been developed. These include Column chromatography, High performance liquid chromatography (HPLC), Gas chromatography, Size exclusion chromatography, Ion exchange chromatography etc. In this book contains more details about the applications of chromatography by various research findings. Each and every topics of this book have included lists of references at the end to provide students and researchers with starting points for independent chromatography explorations. I welcome comments, criticisms, and suggestions from students, faculty and researchers.

\title{
How to reference
}

In order to correctly reference this scholarly work, feel free to copy and paste the following:

Hakan Göker, Maksut Coşkun and Gülgün Ayhan-Kılcıgil (2012). Chromatographic Separation and Identification of Sildenafil and Yohimbine Analogues Illegally Added in Herbal Supplements, Chromatography and Its Applications, Dr. Sasikumar Dhanarasu (Ed.), ISBN: 978-953-51-0357-8, InTech, Available from: http://www.intechopen.com/books/chromatography-and-its-applications/chromatographic-seperation-andidentification-of-a-sildenafil-and-yohimbine-analogue-illegally-added

\section{INTECH}

open science | open minds

\author{
InTech Europe \\ University Campus STeP Ri \\ Slavka Krautzeka 83/A \\ 51000 Rijeka, Croatia \\ Phone: +385 (51) 770447 \\ Fax: +385 (51) 686166 \\ www.intechopen.com
}

\author{
InTech China \\ Unit 405, Office Block, Hotel Equatorial Shanghai \\ No.65, Yan An Road (West), Shanghai, 200040, China \\ 中国上海市延安西路65号上海国际贵都大饭店办公楼 405 单元 \\ Phone: $+86-21-62489820$ \\ Fax: +86-21-62489821
}


(C) 2012 The Author(s). Licensee IntechOpen. This is an open access article distributed under the terms of the Creative Commons Attribution 3.0 License, which permits unrestricted use, distribution, and reproduction in any medium, provided the original work is properly cited. 